\title{
RPA Description of the Electric Polarizability of the Nucleon
}

\author{
J. Geiss, S. Hardt, H. Lenske and U. Mosel \\ Institut für Theoretische Physik, Universität Giessen ${ }^{\dagger}$ \\ D-35392 Giessen, Germany
}

October 11, 2018

\begin{abstract}
Excited states of the nucleon are described as RPA configurations on a mean-field ground state taken from the MIT bag model. A residual interaction of a structure as in the Nambu-Jona-Lasinio model is used. The particle-hole states are coupled to good total angular momentum and isospin. Valence excitations of particle-hole type and quark-antiquark $(q \bar{q})$ states from the Dirac-sea are included. The dependence of the baryon spectrum and multipole response functions on the coupling constant $G$ is studied. At critical values of $G$ the $3 \mathrm{q}^{-}$ ground state becomes degenerate with strongly collective $q \bar{q}$ modes. The model is used to calculate the electric polarizability of the neutron $\alpha_{N}$. Without residual interaction $\alpha_{N}=7 \cdot 10^{-4} \mathrm{fm}^{3}$ is found. With residual interaction the value increases to $\alpha_{N}=(7-11) \cdot 10^{-4} \mathrm{fm}^{3}$. The measured value of $\alpha_{N}$ is reproduced within experimental error bars.
\end{abstract}

${ }^{\dagger}$ Supported by GSI and BMBF 


\section{Introduction}

The electromagnetic polarizabilities are fundamental properties of manybody systems. They are a direct measure for the response of a bound system to an external perturbation and therefore provide direct information on the internal structure and dynamical laws of the system. From recent Compton scattering experiments [1, 2, 3] the polarizability of the neutron $\alpha_{N}=(12 \pm 1.5 \pm 2) \cdot 10^{-4} \mathrm{fm}^{3}$ [2] was extracted. Initiated by these measurements considerable effort has been spent over the last years on theoretical investigations of the polarizability. Since solutions of lattice QCD for finite systems are at a very early stage the theoretical calculations have to rely on effective hadronic models. Reponse functions are a sensitive measure for the dynamical model used to approximate the fundamental QCD interactions. Since the electromagnetic polarizability is determined by processes of low energy-momentum transfer such investigations are suitable extensions from investigations of the ground state to the baryon spectrum at a couple of hundred $\mathrm{MeV}$ of excitation energy. Studies of the polarizability therefore provide a possibility to test the physical content of effective quark models of low energy QCD. In the past calculations have been performed in the Skyrme model [4, 5, 6], the MIT-Bag model [7, 8] and its chiral extensions [9, 10], chiral soliton models [11, 12, 13], chiral perturbation theory [14] and non-relativistic constituent quark models [15]. With quark degrees of freedom only the polarizability is found to range between $\alpha_{N} \sim 8 \cdot 10^{-4} \mathrm{fm}^{3}$ in the MIT-Bag model to $\alpha_{N} \sim 30 \cdot 10^{-4} \mathrm{fm}^{3}$ in the non-relativistic quark models where a quark core root-mean-square (rms) radius of about $\left\langle r^{2}>_{\text {core }}^{1 / 2} \sim 0.7 \mathrm{fm}\right.$ was used. In the chiral quark models where also mesonic degrees of freedom are taken into account the electric polarizability is found to be dominated by the response of the pion cloud to the electric field. In [9] a value of $\alpha_{N} \sim(8-10) \cdot 10^{-4} \mathrm{fm}^{3}$ is obtained for $\left\langle r^{2}>_{\text {core }}^{1 / 2} \sim 0.6 \mathrm{fm}\right.$ and the quark core contributes only about $10 \%$ to $\alpha_{N}$.

At present the theoretical development is at a rather early stage and the calculations have to be considered as exploratory. Very often, wave functions are described in a rather restricted model space containing only a few configurations and interactions are treated perturbatively. The question arises to what extent a more complete description of residual interactions will affect the results. In a strongly interacting system like a hadronic state in the low energy-momentum regime dynamical correlations should be taken into 
account. In a many-body system they can give rise to qualitatively new phenomena which are characterized by phase coherence of many components. Wellkown examples are the giant resonances in nuclei which are observed as strongly collective transitions in response functions. Very likely, coherent motion and collectivity is also important in hadron physics as indicated, for example, by results of the Nambu-Jona-Lasinio (NJL) model [16, 27]. In NJL model mesons are obtained as coherent $q \bar{q}$ excitations of the vacuum [19, 31] and the nucleon was described as a solitonic solution of the field equations [28, 29].

In this paper a first attempt is made to investigate dynamical correlations in a baryon of finite size. In the first place we are interested in global properties of the nucleon close to the ground state rather than in details of baryon spectroscopy. In this energy-momentum region confinement is the prevailing mechanism. Because at present a theory of confinement is still missing we treat confinement in a phenomenological way by using an effective field theory. As discussed in section 2.1 the model follows closely the NJL approach and includes quark fields only. Confinement is enforced by an auxiliary scalar potential chosen as in the MIT bag model [22]. The model space includes up $(u)$ and down $(d)$ quarks and the full range of color states, i.e. we use the MIT-Bag model [22] with $S U_{f}(2)$ flavor and $S U_{c}(3)$ color symmetry. The nucleon ground state is chosen as a 3 -quark $(3 q)$ configuration on top of the filled Dirac sea. The valence quarks are put into the lowest positive energy eigenstate of the bag Hamiltonian. The ground state wave function is fully anti-symmetrized and color-neutral and carries good total angular momentum and isospin. The bag parameters are chosen such that ground state properties as for example the root-mean-square (rms) radius of the nucleon are reproduced.

In section 2.2 excited states of the nucleon and response functions are considered. We are interested only in the changes of wave functions relative to the ground state rather than in a calculation in absolute terms. This allows to use the particle-hole picture which is well established in many-body theory [24, 25] and simplifies the theoretical and numerical effort considerably. The excited states are expanded into one particle-one hole $(1 p 1 h)$ type excitations of the valence shell and $q \bar{q}$ excitations from the Dirac sea into positive energy states. Both types of excitations are coupled to good total internal angular momentum and isospin and, by construction, carry boson quantum numbers. The $q \bar{q}$ contributions are of genuine field theoretical character and are absent 
in a non-relativistic description as e.g. in ref.[32].

Dynamical correlations of ph-states are investigated by introducing an effective residual quark-quark interaction of the NJL-type [16]. The interaction is invariant with respect to Lorentz transformations, rotations in color and flavor space and with respect to chiral transformations. Thus, the relevant symmetries of the QCD are accounted for. Certain matrix elements of the residual interaction are found to be of the order of the unpertubed excitation energies. Thus, a pertubative treatment as chosen in other approaches [17] is inappropriate. Using relativistic RPA theory residual interactions are included to all orders by exact diagonalization within the model space. Moreover, the RPA description also accounts for ground state correlations [23, 24, 25, 26] which are known to be especially important for a reliable description of collectivity.

Relativistic RPA theory is by itself a rather new field. Applications to finite systems exist only for few cases in nuclear structure theory 20, 21. In section 2.3 we present a brief summary of the Dirac RPA formalism and the application to baryonic excitations.

Our main interest is to study the response of baryons to probes with low energy-momentum transfer. From the experience with nuclear response functions it can be expected that correlations and possibly other many-body effects will become observable. Of particular interest are collective states which may lead to coherent excitations with mesonic quantum numbers. This allows to study the evolution of mesonic modes as dynamically correlated $q \bar{q}-$ excitations in a baryonic system.

In section 3 numerical calculations and the application of the approach to the polarizability of the nucleon are presented. In section 3.1 the dependence of the energy spectrum on the model used for the ground state and the residual interaction is discussed. The electrostatic polarizability of the nucleon is investigated in section 3.2. The polarizability is determined from the inverse energy weighted RPA sum rule. Vacuum polarization requires a special treatment. For a comparison to the measured polarizability the physical value of $\alpha_{N}$ is obtained by a RPA calculation including excitations of the valence shells and the Dirac sea from which the polarizability of the bare vacuum is subtracted. The latter is obtained by an independent RPA calculation without valence quark states. In this paper we are mainly interested in the electric polarizability of the neutron. We find that $\alpha_{N}$ is increasing when switching on the residual interaction. We obtain for a quark core root- 
mean-square radius (rms) of about $0.65 \mathrm{fm} \alpha_{N} \approx 7 \cdot 10^{-4} \mathrm{fm}^{3}$ for the pure MIT-Bag in agreement with [9] and $\alpha_{N} \approx 10.5 \cdot 10^{-4} \mathrm{fm}^{3}$ when including the residual interaction. The paper closes in section 4 with a summary and concluding remarks.

\section{Effective Theory of Hadron Structure}

\subsection{Nucleon Ground State and Effective Hamiltonian}

In this section an effective quark field theory of a dynamical structure similar to the Nambu-Jona-Lasinio (NJL) model [16] is discussed. The NJL model can be considered as a model for the quark sector of QCD. Gluonic contributions are integrated out. They are treated schematically in terms of a chirally symmetric two-body contact interaction. Of special interest for hadron physics is that chiral symmetry is spontaneously broken in the NJL model. For coupling constants beyond a critical value a sudden transition from massless quarks to heavy constituent quarks is observed. Thus, the NJL model explains the chiral phase transition dynamically. It is also well suited for model studies of dynamical correlations [19, 27], medium effects and solitonic states [28, 29]. A review of the NJL model and applications is found in ref.[27]. For our purpose we use a model Lagrangian $\mathcal{L}$

$$
\begin{aligned}
\mathcal{L} & =\mathcal{L}_{M F}+\mathcal{L}_{i n t} \\
\mathcal{L}_{M F} & =\bar{\Psi}\left(i \gamma_{\mu} \partial^{\mu}-m_{q}-U_{s}\right) P s i \\
\mathcal{L}_{\text {int }} & =\mathcal{L}_{\text {int }}^{c}+\bar{\Psi} U_{s} \Psi \\
\mathcal{L}_{\text {int }}^{c} & =G \sum_{i=1}^{8}\left(\bar{\Psi} \gamma_{\mu} \frac{\lambda^{i}}{2} \Psi\right)^{2} .
\end{aligned}
$$

given by a mean-field part $\mathcal{L}_{M F}$ and a residual interaction including a twobody color-color interaction $\mathcal{L}_{\text {int }}^{c}$. An auxiliary scalar potential $U_{s}$ has been introduced in order to obtain confinement. In practice confinement is described in the MIT bag model which means to set $U_{s}=$ const. inside the bag and to impose appropriate confining boundary conditions on the bag surface. The quark current mass is denoted by $m_{q}$.

The interactions $\mathcal{L}_{\text {int }}^{c}$ is given by a scalar coupling of color currents. The color structure is determined by the generators $\lambda_{i}, i=1 \cdots 8$ of $S U_{c}(3)$ [30]. 
In a color neutral state the matrix elements of such an interaction are completely determined by the exchange contributions. For a contact interaction as in $\mathcal{L}_{\text {int }}^{c}$ an equivalent direct interaction is obtained after a Fierz transformation [30] which for $S U_{f}(2)$ is given by

$$
\mathcal{L}_{\text {int }}^{c}=\sum_{k=S, V \cdots}\left(a_{k}\left(\bar{\Psi} \Gamma_{k} \Psi\right)^{2}+b_{k}\left(\bar{\Psi} \tau \Gamma_{k} \Psi\right)^{2}\right)
$$

where $\Gamma_{k}=1, \gamma_{\mu} \cdots$ is a scalar, vector etc. Dirac operator and $\boldsymbol{\tau}$ is a Pauli isospin operator. The coefficients $a_{k}$ and $b_{k}$ are related to the coupling constant $G$ by the elements of the Fierz transformation matrix.

The stationary solutions to the mean-field Lagrangian $\mathcal{L}_{M F}$ are given by the eigenstates of the Hamiltonian $H_{M F}=H_{b a g}$

$$
\left[\boldsymbol{\alpha} \boldsymbol{p}+\beta M(\boldsymbol{r})-E_{n, \kappa}\right] \Psi_{n, \kappa, j}(\boldsymbol{r})=0
$$

where confinement is described by the effective mass operator

$$
M(\boldsymbol{r})=m_{q}+U_{s}(\boldsymbol{r})=\left\{\begin{array}{cc}
M_{0} ; & r<R \\
\infty ; & r>R
\end{array}\right.
$$

and the constant $M_{0}$ can be set to zero. $\kappa, j$ are the eigenvalues of the operators $K=\beta(\boldsymbol{\Sigma} \cdot \boldsymbol{\ell}+1)$ and the total angular momentum $\boldsymbol{j}=\boldsymbol{\ell}+\boldsymbol{s}$. The quark wave functions can be written in the form

$$
\Psi_{n, \kappa, j}=\left(\begin{array}{r}
g_{n, \kappa}(r) \mathcal{Y}_{j l}^{m} \\
-i f_{n, \kappa}(r) \mathcal{Y}_{j l^{\prime}}^{m}
\end{array}\right) e^{-i E_{n, \kappa} t} .
$$

Quantization is obtained by the condition that the quark currents vanish at the surface of the bag [22]. In $S U_{f}(2)$ the lowest positive energy level has quantum numbers $j^{\pi}=\frac{1}{2}^{+}, t=\frac{1}{2}$ and is therefore fourfold degenerate. The ground state of a baryon is built of three quarks in the valence shell which are coupled to good total angular momentum and parity $J^{\pi}$ and isospin $T$. In addition, the state must be a $S U_{c}(3)$ color singlet. In second quantization the wave function of a neutron with spin projection $m_{j}=+\frac{1}{2}$ is given by [33]

$$
|n \uparrow\rangle=\frac{1}{\sqrt{18}} \sum_{\alpha \beta \gamma=1}^{4} T_{\alpha \beta \gamma} a_{\alpha}^{1^{\dagger}} a_{\beta}^{2 \dagger} a_{\gamma}^{3^{\dagger}}|0\rangle .
$$


The bare vacuum is denoted by $|0\rangle$ and the states in color space are labeled by $1,2,3$. The summations over $\alpha, \beta, \gamma$ account for the two flavors and the spin projections where indices have been assigned

$$
|u \uparrow\rangle \mapsto 1 \quad|u \downarrow\rangle \mapsto 2 \quad|d \uparrow\rangle \mapsto 3 \quad|d \downarrow\rangle \mapsto 4
$$

The coefficients $T_{\alpha \beta \gamma}$ are given by

$$
T_{\alpha \beta \gamma}=\left\{\begin{array}{rlll}
-1 ; & (\alpha, \beta, \gamma)=(134)+\text { permutations } \\
2 ; & (\alpha, \beta, \gamma)=(233)+\text { permutations } \\
0 ; & \text { otherwise. }
\end{array}\right.
$$

From the energy-momentum tensor $T^{\mu \nu}$ the ground state energy is obtained as

$$
E_{g s}=\left\langle n\left|T^{00}\right| n\right\rangle=\sum_{k} E_{k} g(k)+\left\langle n\left|\bar{\Psi} U_{s} \Psi\right| n\right\rangle+\left\langle n\left|\mathcal{L}_{\text {int }}\right| n\right\rangle
$$

where the mean-field energies $E_{k}$ are weighted by the ground state occupation probabilities

$$
\left\langle n \uparrow\left|a_{k}^{\dagger} a_{k^{\prime}}\right| n \uparrow\right\rangle=\delta_{k, k^{\prime}} \cdot g(k)=\delta_{k, k^{\prime}} \cdot\left\{\begin{aligned}
1, & E_{k}<0 \\
0, & E_{k}>E_{v a l} \\
\frac{1}{18} g_{v}(k), & E_{k}=E_{v a l}
\end{aligned}\right.
$$

and

$$
g_{v}(1)=2 \quad g_{v}(2)=4 \quad g_{v}(3)=10 \quad g_{v}(4)=2
$$

where the same labeling as in Eq.(10) is used.

The use of the phenomenological potential $U_{s}$ clearly describes confinement in a non-self-consistent way. Translational and chiral symmetry is broken by $U_{s}$ and from Hartree-Fock theory it is known that this may lead to admixtures of spurious states into the spectrum. This occurs in channels with the same quantum numbers as the broken symmetry, for example the spurious centre of mass motion in the isoscalar $J^{\pi}=1^{-}$channal. By imposing the condition that the residual interaction does not contribute to the ground state the depth of the confining potential or equivalently the constant $M_{0}$, Eq.(7), can be fixed

$$
U_{s}=-2 a_{s}\langle n|\bar{\Psi} \Psi| n\rangle
$$


where $a_{s}$ is the scalar coupling constant after the Fierz transformation. By this relation self-consistency between the auxiliary potential $U_{s}$ and the twobody interaction is restored at least at an average.

After the ground state has been obtained we next consider excited states of the nucleon. For that purpose it is convenient to extract an effective Hamiltonian from the energy-momentum tensor. We arrive at a form which can be written as

$$
H=\sum_{k} E_{k} a_{k}^{\dagger} a_{k}+\frac{1}{4} \sum_{p q r s} V_{p r q s}: a_{p}^{\dagger} a_{r}^{\dagger} a_{s} a_{q}:
$$

where $E_{k}$ are the MIT-bag eigenenergies and the normal ordering is taken with respect to the nucleon groundstate. The matrix elements of the residual interaction are defined by

$$
V_{p r q s}=G \int d^{3} r\left(\bar{\varphi}_{p} \gamma_{\mu} \frac{\lambda_{\alpha}}{2} \varphi_{q}\right)\left(\bar{\varphi}_{r} \gamma^{\mu} \frac{\lambda_{\alpha}}{2} \varphi_{s}\right)
$$

The summations include contributions from positive and negative energy states. An energy cut-off $\Lambda$ in the ph-space has to be introduced. This corresponds to the fact, that the interaction of Eq.(17) would have to be regularized in case the mean-field had been extracted self-consistently. A cut-off is also necessary by numerical reasons in order to keep the calculations manageable.

\subsection{Excited States and Response Functions}

An appropriate approach to small amplitude oscillations is to represent excited states of the nucleon by particle-hole excitations with respect to the ground state. This means to use the ground state as a new vacuum and to assume that the wave functions of excited states are mainly determined by a redistribution of single particle strength from occupied into initially empty states. In a relativistic system this redistribution process also includes transitions from the Dirac sea into positive energy states. The ground state might contain admixtures of many-body configurations beyond the simple mean-field state. In that case excited states also include components from the annihilation of such ground state correlations [23, 26]. In RPA theory 
this is taken into account by using state operators

$$
Q_{\nu}^{\dagger}\left(J, M, T, M_{T}\right)=\sum_{p h}\left[X_{p h}^{\nu} A_{p h}^{\dagger}\left(J, M, T, M_{T}\right)-Y_{p h}^{\nu} A_{p h}\left(\bar{J}, M, \bar{T}, M_{T}\right)\right] .
$$

which include $1 p 1 h$ excitation operators

$$
A_{p h}^{\dagger}\left(J, M, T, M_{T}\right)=\left(a_{p}^{\dagger} \otimes a_{h}\right)_{T, M_{t}}^{J, T}
$$

and annihilation operators

$$
A_{p h}\left(\bar{J}, M, \bar{T}, M_{T}\right)=(-1)^{J+M+T+M_{t}}\left(a_{h}^{\dagger} \otimes a_{p}\right)_{T,-M_{t}}^{J,-M} .
$$

The particle and hole operators $a_{p}^{\dagger}$ and $a_{h}$ are coupled to total angular momentum $J$ and isospin $T$ with projections $M$ and $M_{T}$, respectively. The structure of the RPA state operators and their dynamics will be discussed in the next section. Here, we consider first their application to excited states of the nucleon. Denoting the ground state by $\left|j_{0} m_{0}\right\rangle$ excited states are given in general by a superposition of RPA multipole operators

$$
\left|j m, t t_{3}\right\rangle=\sum_{J, T}\left[Q^{\dagger}(J, T) \otimes\left|j_{0}, t_{0}\right\rangle\right]_{j m, t t_{3}}
$$

Since the RPA operators carry bosonic quantum numbers it is seen that excited states are actually expressed by a superposition of valence excitations and meson-like modes. The wave functions of Eq.(21) lead to the so-called tensor RPA [18] which will not be discussed here. An important simplification is obtained if only multipole response functions and sum rules are considered. In that case one is only interested in the total strength for a transition of a given multipolarity regardless of the spin states $\left(j m, t t_{3}\right)$ which were participating in the excitation process. This amounts to average over the spin and isospin projections of the initial state $\left(j_{0} m_{0}, t_{0} t_{30}\right)$ and to sum over final spin and isospin quantum numbers $\left(j m, t t_{3}\right)$. The spin averaging corresponds to project out the bosonic RPA modes of the same tensorial rank as the operator of the external probe. In electric dipole transitions, for example, only the $J^{\pi}=1^{-}$components of the final states contribute. Therefore, spin averaged response functions and sum rules are described to a good approximation already in the uncoupled representation

$$
\left|j_{0} m_{0}, t_{0} t_{30} ; J M, T M_{T}\right\rangle=Q^{\dagger}\left(J M, T M_{T}\right)\left|j_{0} m_{0}, t_{0} t_{30}\right\rangle
$$

and these wave functions will be used in the following. 


\subsection{Dirac RPA Theory}

The RPA state operators, Eq.(18), are of a particular structure which goes beyond a pure mean-field description. The terms containing the annihilation operators correspond to processes which propagate backward in time. Such processes only contribute if the ground state includes correlations of $2 p 2 h$ or higher order. The correlated RPA ground state $\left|0_{c}\right\rangle$ is defined by the orthogonality condition

$$
Q_{\nu}\left|0_{c}\right\rangle=0
$$

which must be fulfilled for all RPA states $\nu$. As shown in ref. 26] the RPA and the mean-field ground states are related by

$$
\left|0_{c}\right\rangle=e^{S}|0\rangle
$$

where correlations are introduced by the operator

$$
S=\sum_{p h, p^{\prime} h^{\prime}, k k_{z}} C_{p h p^{\prime} h^{\prime}}\left(k, k_{z}\right) A_{p h}^{\dagger}\left(k, k_{z}\right) A_{p^{\prime} h^{\prime}}^{\dagger}\left(k,-k_{z}\right)
$$

from which the many-body structure of $\left|0_{c}\right\rangle$ is apparent. For a shorthand notation the combined spin-isospin quantum numbers $\left(k, k_{z}\right)=\left(J M, T M_{T}\right)$ were introduced. Because the correlation matrix $C$ is defined in terms of the "backward" amplitudes $Y$ [26] RPA can be solved self-consistently by successive recalculation of the ground state. However, here we follow the common practice and determine the $Y$-amplitudes in first approximation which corresponds to the quasi-boson approximation (QBA). It means to retain in Eq.(24) the lowest order term in $S$ when matrix elements and occupation probabilities involving the time-reversed state operators are to be evaluated.

In relativistic RPA the ground state correlations also include $q \bar{q}$ admixtures from virtual excitations of the vacuum. In nuclei these contributions are suppressed to a large extent [20, 21] because of the large mass gap. A qualitatively different situation is encountered in quark models of hadrons. Klimt et al. [19] have used the NJL model to calculate the meson states below $1 \mathrm{GeV}$ as collective $q \bar{q}$ excitations of the vacuum. From the results of ref.[19] it can be expected that $q \bar{q}$ vacuum correlations are of importance also for baryons. Physically, they describe the dressing of the quark core by a virtual meson cloud due to dynamical vacuum polarization. 
The RPA state operators describe stationary eigenstates of the full Hamiltonian, Eq.(16). They are determined by the equation of motion [23, 24]

$$
\left[H, Q_{\nu}^{\dagger}\right]=\omega_{\nu} Q_{\nu}^{\dagger}
$$

where $\omega_{\nu}=E_{\nu}-E_{g s}$ is the excitation energy. Taking the commutator from the left with resepct to the $1 p 1 h$ excitation and annihilation operators, Eqs.(19) and (20), the RPA eigenvalue equation

$$
\left(\begin{array}{cc}
A\left(k, k_{z}\right) & B\left(k, k_{z}\right) \\
B^{*}\left(k,-k_{z}\right) & A^{*}\left(k,-k_{z}\right)
\end{array}\right)\left(\begin{array}{l}
X^{\nu} \\
Y^{\nu}
\end{array}\right)=\omega_{\nu}\left(\begin{array}{cc}
N & 0 \\
0 & N
\end{array}\right)\left(\begin{array}{l}
X^{\nu} \\
Y^{\nu}
\end{array}\right)
$$

is obtained from which $\omega_{\nu}$ and the state vectors $\left(X_{\nu}, Y_{\nu}\right)$ are derived. The matrix elements are defined by ground state expectation values of double commutators

$$
\begin{gathered}
A_{m i, n j}\left(k, k_{z}\right)=\left\langle 0\left|\left[A_{m i}\left(k, k_{z}\right),\left[H, A_{n j}^{\dagger}\left(k, k_{z}\right)\right]\right]\right| 0\right\rangle \\
B_{m i, n j}\left(k, k_{z}\right)=-\left\langle 0\left|\left[A_{m i}\left(k, k_{z}\right),\left[H, A_{n j}\left(\bar{k}, k_{z}\right)\right]\right]\right| 0\right\rangle
\end{gathered}
$$

and the metric is given by

$$
\begin{aligned}
N_{m i, n j}\left(k, k_{z}\right) & =\left\langle 0\left|\left[A_{m i}\left(k, k_{z}\right), A_{n j}^{\dagger}\left(k, k_{z}\right)\right]\right| 0\right\rangle \\
\bar{N}_{m i, n j}\left(k, k_{z}\right) & =\left\langle 0\left|\left[A_{m i}^{\dagger}\left(\bar{k}, k_{z}\right), A_{n j}\left(\bar{k}, k_{z}\right)\right]\right| 0\right\rangle .
\end{aligned}
$$

$m, n(i, j)$ denote single quark states above (below) the Fermi-surface.

Evaluating the commutators the metric $N$ is found to be given by the expectation values

$$
\begin{aligned}
& N_{m i, n j}=\left\langle 0\left|\delta_{i, j} \delta_{m, n}-\delta_{i, j} a_{n}^{\dagger} a_{m}-\delta_{m, n} a_{j} a_{i}^{\dagger}\right| 0\right\rangle \\
& \bar{N}_{m i, n j}=\left\langle 0\left|-\delta_{i, j} \delta_{m, n}+\delta_{i, j} a_{n}^{\dagger} a_{m}+\delta_{m, n} a_{j} a_{i}^{\dagger}\right| 0\right\rangle .
\end{aligned}
$$

In an open shell system like the nucleon where the valence shell is only partially occupied the metric is determined by the ground state occupation probabilities $g(k)$, Eq. (13),

$$
N_{m i, n j}=-\bar{N}_{m i, n j}=\delta_{i, j} \delta_{m, n}(1-g(i)-g(m)) .
$$

In a system with a fully occupied valence shell the contributions from the normal ordered operator parts vanish and the standard RPA metric is retrieved:

$$
N_{m i, n j}=-\bar{N}_{m i, n j}=\delta_{i, j} \delta_{m, n} .
$$




\section{Results and Discussion}

The model accounts for a number of important features of hadron structure physics, at least in the quark sector, and it is therefore meaningful to calculate observables and compare them to data. In view of the phenomenological description of confinement, the schematic ansatz for the residual interaction and the restricition to the quark sector we consider the results as exploratory. However, finite size effects are taken into account and RPA theory allows to study residual interactions in a relativistic invariant formulation.

\subsection{Energy Spectrum}

The ground state of the nucleon was described in MIT bag model where a bag radius $R_{b a g}=0.9 \mathrm{fm}$ was used which corresponds to a rms radius $\left\langle r^{2}\right\rangle_{c}^{1 / 2}=$ $0.65 \mathrm{fm}$ for the quark core. A value less than the empirically known proton charge radius $(0.8 \mathrm{fm})$ is used because the model clearly does not account for contributions from the meson cloud surrounding the core. The spectrum of $1 p 1 h$ states obtained in the bag model is known to give a too high level density at low excitation energy [17] and, even more severe, the lowest excited states are of negative parity (see Fig.1). This is in clear contradiction to experiment where the positive parity $\Delta(1232)$ resonance is found as the first excited state above the nucleon. In terms of the transferred angular momentum, as introduced in section 2.2 , this is a $J^{\pi}=1^{+}$"pionic" excitation on the nucleon ground state. Within the bag model a more realistic level sequence could not be obtained. The results simply reflect the properties of the spectrum of an infinite well where the orbits group into shells of alternating parity. Spectra obtained with confining potentials of different shapes, e.g. linearily increasing with radius, led to similar results. As it might have been expected a bag model accounts only in a very rough way for the dynamics of quarks in a nucleon.

The question arises whether the residual interaction could improve on this situation. In the RPA calculations excitations from the valence shell and the Dirac sea with unperturbed energies up to $2200 \mathrm{MeV}$ where used which corresponds to configuration space of about $N=40$ unpertubed $1 p 1 h$ and $q \bar{q}$ states. The dependence of the RPA eigenenergies on the coupling constant $G$ of the residual interaction, Eq.(四), is illustrated in Fig.1 for isoscalar $J^{\pi}=0^{ \pm}$ and isovector $J^{\pi}=1^{-}$states. Since these channels involve spin and isospin 
modes, respectively, they are free of admixtures from spurious translational states. The special form of the residual interaction leads after the Fierz transformation to isoscalar $(T=0)$ and isovector $(T=1)$ interactions of the same structure and interaction strengths. Thus, for given $J^{\pi}$, isoscalar and isovector excitations behave in the same way when $G$ is varied and it is sufficient to consider only one of the two channels.

Over a wide range of interaction strengths the eigenenergies remain on their unperturbed values and seem to be almost unaffected by the residual interaction. An inspection of the RPA matrix shows that the nondiagonal elements of the RPA matrix are rather large and may reach values of the order of the lowest $p h$ energies. Thus, strong cancellations must occur which compensate the contributions from the diagonal matrix elements. For $G>6 \cdot 10^{-6} \mathrm{MeV}^{-2}$ the first $J^{\pi}=0^{-}$state is lowered and approaches the ground state $(\omega=0)$ at $G_{\text {crit. }}=6.8 \cdot 10^{-6} \mathrm{MeV}^{-2}$. At even larger values of $G$ the energy becomes purely imaginary which is a wellknown property of the RPA [23]. On the way down to zero excitation energy the states gains collectivity and an increasing number of configurations contribute. When become degenerate with the ground state the ground state correlations strongly increase which is seen from the fact that the $Y$ and $X$ amplitudes are of the same magnitude. Close to this value of $G$, however, the RPA ceases to be valid and, as discussed in section 2.3, an improved description of the RPA ground state would be necessary. These transition depends to some extend on the multipolarity. In Fig. 1 also results for the $J^{\pi}=0^{+}$channel are shown. In that case $G_{\text {crit }}=6.4 \cdot 10^{-6} \mathrm{MeV}^{-2}$ is found. In the $J^{\pi}=1^{-}$channel the cancellation effects extend to larger coupling constants. As a result the states are less affected by residual interactions in the region of $G$ values shown in Fig.1.

The results lead to the conclusion that a perturbative treatment of residual interactions in a relativistic system as e.g. in ref. [17] might be unreliable. The RPA results show that an exact diagonalization can lead to cancellations such that even if the matrix elements are individually large the net effect is minor.

Calculations with larger configuration spaces led to qualitatively the same results. The dependence on $G$ roughly scales with the size of the configuration space. The results indicate that the product of $\mathrm{G}$ and the square of the cut-off energy $\Lambda$ is close to a constant. A similar scaling law is found in standard NJL calculations with a three- or four-momentum cut-off $\Lambda_{p}$ where observables 
depend only on $G \Lambda_{p}^{2}$.

\subsection{The Electromagnetic Polarizability}

The electric and magnetic polarizabilities $\alpha$ and $\beta$, respectively, are a measure for the response of a system to a time independent external electromagnetic field. Theoretically, they are defined by the inverse energy weighted sum rule [34]

$$
\alpha=2 \cdot \sum_{\nu \neq 0} \frac{\left|\left\langle 0\left|D_{z}\right| \nu\right\rangle\right|^{2}}{E_{\nu}-E_{0}}
$$

where the ground state and the RPA excited states are denoted by $|0\rangle$ and $|\nu\rangle$, respectively. The transition operator is obtained by minimal coupling of the quark fields to the electromagnetic field. The electric polarizability is determined by the dipole operator

$$
\begin{aligned}
D_{z} & =e \int \bar{\Psi} \gamma_{0} A_{0} \Psi d^{3} r \\
A_{0} & =z=\sqrt{\frac{4 \pi}{3}} r Y_{1}^{0}
\end{aligned}
$$

For the nucleon the RPA matrix elements of $D_{z}$ are given by

$$
\langle n|D| \nu\rangle=\sum_{p h}\langle p|D| h\rangle\left(X_{p h}^{\nu}+Y_{p h}^{\nu}\right)(g(h)-g(p))
$$

The angular momentum and isospin coupled matrixelement of $D_{z}$ is denoted by $\langle p|D| h\rangle$. The summation includes $1 p 1 h$ valence and $q \bar{q}$ vacuum excitations. $g$ are the occupation numbers, Eq.(13), averaged over the spin projections $M$. From the transition matrix elements it is seen that the structure of the RPA wave functions is directly tested by the polarizability.

A special feature of relativistic systems is vacuum polarization. The contributions corresponding to $q \bar{q}$ excitations of the bare vacuum have to be removed from Eq.(33) before a quantity is obtained which can be compared to data. The physical polarizability is determined by subtracting the polarizability $\alpha_{v a c}$ of the bare vacuum from the polarizability $\alpha_{v a c+3 q}$ of the full system,

$$
\alpha_{N}=\alpha_{v a c+3 q}-\alpha_{v a c}
$$


Both values $\alpha_{v a c+3 q}$ and $\alpha_{v a c}$ are obtained by RPA calculations on the bag groundstate.

In Fig. $2 \alpha_{v a c+3 q}$ and $\alpha_{v a c}$ are shown as a function of $G$. Both values are seen to increase with the strength of the residual interaction. This behaviour reflects the gain in collectivity with increasing interaction strength which is typical for RPA response functions. A collective state is obtained when a large number of basis states are phase coherent. A special property of the RPA is the additional increase in transition strength from the ground state correlations. In a collective state the $y$ and $x$ amplitudes carry the same sign and Eq.(36) shows that matrix elements will considerably be enhanced. The effect is especially pronounced for low-lying state which are close to the ground state and thus are most strongly affected by ground state correlations.

At larger $G$ collectivity becomes important in $\alpha_{v a c+3 q}$ as seen from the increasing separation between the two curves in Fig.2. Apparently, the increase in collectivity is stronger for the valence components than for the vacuum excitations. The effect is seen more clearly in $\alpha_{N}$, displayed in the lower part of Fig.2. Over the range of coupling constants $\alpha_{N}$ increases by about $50 \%$. The gain in transition strength indicates the gradual built-up of coherence in the RPA wave functions and the valence excitations participate more strongly than the $q \bar{q}$ configurations. This result shows that the electrostatic polarizability is a measure for the properties of the quark core being less sensitive to vacuum effects. For $G>3 \cdot 10^{-6} \mathrm{MeV}^{-2}$. the theoretical results range within the error band of the measured value $\alpha_{N}=(12 \pm 1.5 \pm 2) \cdot 10^{-4} \mathrm{fm}^{3}$ [2]. Thus the observed polarizability is reproduced reasonably well.

\section{Summary and Conclusions}

An effective field theoretical model was used to study dynamical correlations in excited states of the nucleon. Starting from a Lagrangian of a structure similar to the NJL model an effective Hamiltonian was derived. Confinement was introduced by means of an phenomenological scalar potential. In applications the MIT bag model was used. The ground state of the nucleon was described by color-singlet wave function with good total angular momentum and isospin. A two-body color-color interaction was used in relativistic RPA calculations on a nucleon ground state. In exploratory model calculations the experimentally known electrostatic polarizibility of the nucleon was studied. 
The empirical value was reasonably well reproduced. Similar to ref. [9] we are led to the conclusion that the coupling of the external photon to mesonic modes which in our approach are represented by collective RPA excitations gives important contributions to the polarizability. The model calculations indicate a special sensitivity of the polarizability to the quark core. The agreement supports at least the physical concept of the model. However, in view of the remaining uncertainties it is hardly possible to draw final conclusions on details of the dynamics and other physical aspects. The results for energy spectra and the polarizability constrain the coupling constant to the region $G=3 \cdots 6 \cdot 10^{-6} \mathrm{MeV}^{-2}$. 


\section{References}

[1] F. Federspiel et al., Phys. Rev. Lett. 67, 1511 (1991)

[2] J. Schmiedmayer et al., Phys. Rev. Lett. 66, 1015 (1991)

[3] A. Ziegler et al., Phys. Lett. B 278, 34 (1992)

[4] I. Zahed and G. E. Brown, Phys. Rep. 142, 1 (1986)

[5] S. Scherer and P.J.Mulders, Nucl.Phys. A 549, 521 (1992)

[6] E. M. Nyman, Phys Lett. B 142, 388 (1984)

[7] P. C. Hecking and G. F. Bertsch, Phys. Lett. B 99, 237 (1981)

[8] A. Schaefer et al., Phys. Lett. B 143, 323 (1984)

[9] R. Weiner and W. Weise, Phys. Lett. B 159, 85 (1985)

[10] V. Bernard et al., Phys. Lett. B 205, 16 (1988)

[11] W. Broniowsky, M. K. Banerjee and T. D. Cohen, Phys. Lett. 283, 22 (1992)

W. Broniowsky and T. D. Cohen, Phys. Rev. D 47, 299 (1993)

E. M. Nikolov, W. Broniowsky and K. Goeke, Nucl. Phys. A 579, 389, (1994)

[12] N. N. Scoccola and W. Weise, Phys. Lett. B 232, 287 (1989)

N. N. Scoccola and W. Weise, Nucl. Phys. A 517, 495 (1990)

[13] M. Chemtop, Nucl. Phys. A 473, 613 (1987)

[14] V. Bernard, N. Kaiser and U. G. Meissner, Phys. Rev. Lett. 67, 1515 (1991)

V. Bernard, N. Kaiser and U. G. Meissner, Nucl. Phys. B 373, 346 (1992)

[15] M Traini and R. Leonardi, Phys. Lett. B 334, 7 (1994)

[16] Y. Nambu and G. Jona-Lasinio, Phys. Rev. 122, 345 (1961) 
[17] T. A. DeGrand, Annals of Physics 101, 496 (1976)

T. A. DeGrand and R. L. Jaffe, Annals of Physics 100, 425 (1976)

[18] D. C. Rowe and C. Ngo-Trong, Rev. Mod. Phys. 47, 471 (1975)

S. Hardt, Hadrons '95 Proceedings, Excitations of the Nucleon within the Random Phase Approximation, World Scientific, Singapore

[19] S. Klimt, M. Lutz, U.Vogl and W. Weise, Nucl. Phys. A 516, 429 (1990)

[20] J. A. McNeil, R. J. Furnstahl, E. Rost and J. R. Shepard, Phys. Rev C 40, 399 (1989)

[21] J. A. McNeil, E. Rost and J. R. Shepard, Phys. Rev C 40, 2320 (1989)

[22] A. Chodos, R. Jaffe, K. Johnson, C. Torn and V. Weisskopf, Phys. Rev D 9, 3471 (1974)

[23] D.J. Rowe, Nuclear Collective Motion, Menthuen, New York, 1966.

[24] A.L. Fetter, S.D. Walecka, Quantum Theory of Many-Particle System, McGraw-Hill, New York, 1971.

[25] J.W. Negele and H. Orland, Quantum Many-Particle System, Addison-Wesley, New York, 1988.

[26] H. Lenske and J. Wambach, Phys.Lett. B 249, 377 (1990).

[27] S.P. Klevansky, Rev.Mod.Phys. 64, 649 (1992).

[28] C. Schüren, F. Döring, E. Ruiz Arriola and K. Goeke, Nucl. Phys A 595, 687, (1993)

[29] R. Alkhofer, H. Reinhardt and H. Weigel, Tübingen preprint No UNITU-THEP-25 (1994)

[30] C. Itzykson, J. Zuber Quantum Field Theory, McGraw Hill, Singapore 1987 
[31] V. Bernard, R. Brockmann, M. Schaden, W. Weise, E. Werner, Nucl. Phys. A 412, 349 (1984)

[32] N. Isgur und G. Karl, Phys. Let. 72 B, Nr. 1, (1977) 109

N. Isgur und G. Karl, Phys. Rev. 72 D, Nr. 11, (1978) 4187

[33] U. Mosel, Quarks, Fields and Symmetries, McGraw Hill, Hamburg 1989

[34] P. Ring, P. Schuck The Nuclear Many-Body Problem, Berlin 1980 


\section{FIGURE CAPTIONS}

Fig. 1.

Energy-spectra in the $J^{\pi}=0^{-}, 1^{-}, 0^{+}$channels as a function of the coupling constant $G$. In all cases only the first five excited states are shown

Fig. 2.

Polarizability of the neutron as a function of the coupling strength of the residual interaction. In the upper part RPA results for the vacuum polarizability $\alpha_{v a c}$ (dashed) and the polarizability $\alpha_{v a c+3 q}$ including valence states (full line ) are shown. In the lower panel the empirical (dashed) and the theoretical polarizability (full line) of the neutron are displayed. Additionally the lower bound of the empirical value is shown (dotted line). 

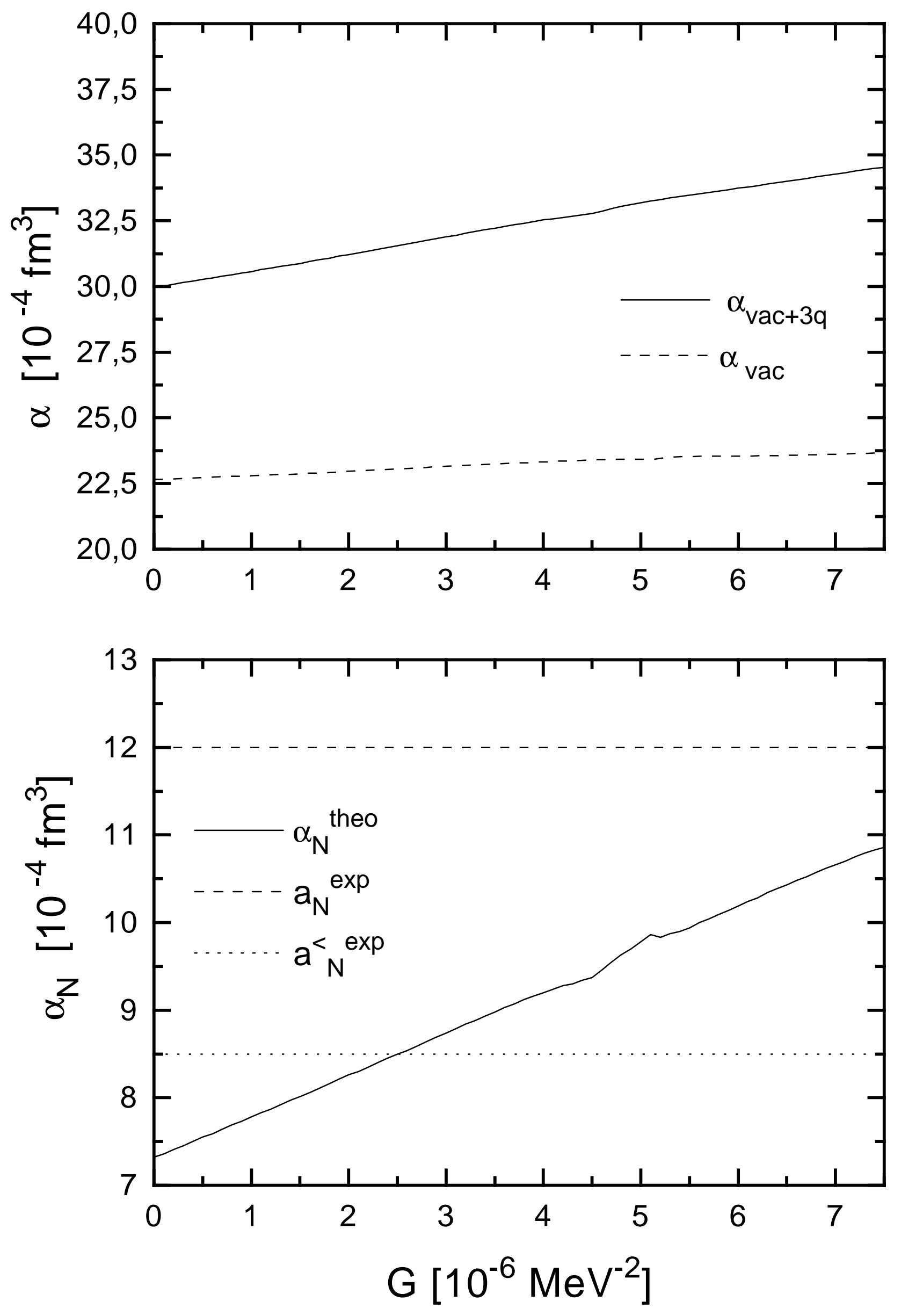